\title{
QUANTUM COLLAPSE, CONSCIOUSNESS AND SUPERLUMINAL COMMUNICATION
}

\author{
Gao Shan \\ The Scientists Work Team of Electro-Magnetic Wave Velocity \\ Chinese Institute of Electronics \\ and \\ Institute of Electronics, Chinese Academy of Sciences \\ LongZeYuan 24-3-501, HuiLongGuan, ChangPing District \\ Beijing 100096, P.R.China \\ E-mail: rg@mail.ie.ac.cn
}

The relation between quantum collapse, consciousness and superluminal communication is analyzed. As we know, quantum collapse, if exists, can result in the appearance of quantum nonlocality, and requires the existence of a preferred Lorentz frame. This may permit the realization of quantum superluminal communication (QSC), which will no longer result in the usual causal loop in case of the existence of a preferred Lorentz frame. The possibility of the existence of QSC is further analyzed under the assumption that quantum collapse is a real process. We demonstrate that the combination of quantum collapse and the consciousness of the observer will permit the observer to distinguish nonorthogonal states in principle. This provides a possible way to realize QSC. Some implications of the existence of QSC are briefly discussed.

KEY WORDS: Quantum collapse, nonlocality, preferred Lorentz frame, consciousness, superluminal communication, special relativity 


\section{QUANTUM COLLAPSE, NONLOCALITY AND PREFERRED LORENTZ FRAME}

The property of quantum nonlocality was first brought to the attention of the physics community by Einstein et al[1]. Later Bell made a big stride forward in the study of quantum nonlocality[2]. The famous Bell theorem shows the contradiction between locality and quantum mechanics, especially the quantum collapse assumption. Many experiments have been conducted to confirm Bell's theorem up to now $[3,4,5,6]$. Although the results basically confirm the predictions of quantum mechanics, and reveal the actual existence of quantum nonlocality, physicists expressed a variety of different opinions about the conclusion that quantum mechanics allow the existence of quantum nonlocality. In Shimony's opinion[7], this indicates that the existing quantum theory can be compatible with special relativity. But Aharonov and Albert pointed out a special difficulty in combining quantum mechanics with special relativity when taking the measurement process as one kind of realistic process[8]. In fact, Bell himself also realized the inconsistency of his theorem with special relativity, and thought that there exists a deeper level which is not Lorentz invariant, hidden behind the apparent Lorentz invariance of the phenomena[9]. Bell suggested that there might exist a preferred Lorentz frame, in which actual causal sequences of nonlocally correlating events can be defined.

Recently, the incompatibility between quantum nonlocality and special relativity has been further demonstrated from different points of view $[10,11,12,13$, 14, 15]. It has been argued that any dynamical theory describing the collapse of the wave function, in which the predictions of the theory agree with those of ordinary quantum theory, must have a preferred Lorentz frame. A general 
demonstration was given by Percival[12]. His conclusion based upon the realistic assumption of the quantum collapse process was that quantum nonlocal phenomena do not satisfy Lorentz invariance, thus resulting in the existence of a preferred Lorentz frame. In other words, the consistent description of quantum nonlocal phenomena requires a preferred Lorentz frame. Since Percival's demonstration was independent of any causality assumption in the quantum domain, and only depended on the causal relation between the classical input and output, his conclusion is universal. It is further guessed that the preferred Lorentz frame may be the cosmos frame, in which the microwave background radiation is isotropic. For detailed discussions please refer to the books[15, 16].

It can be seen that the above conclusion is a special case of a general conclusion, which says that only one speed value is permitted to be invariant in any Lorentz frame[15]. If we assume that the collapse process of the wave functions of the particles in a two-particle entanglement state is simultaneous in all Lorentz frames, i.e. the simultaneity of the collapse of wave function possesses Lorentz invariance, then there will appear two speed values. In this case light speed and infinite speed are both invariant in any Lorentz frame. Thus one of the speed values must be not invariant in all Lorentz frames. This will naturally result in the existence of a preferred Lorentz frame. The standard convention is to stipulate the constancy of one-way light speed, then the simultaneity of the collapse of wave function will not possess Lorentz invariance[17]. A strict physical definition of the preferred Lorentz frame is that in this frame the collapse of wave function happens simultaneously in different positions in space, and the causal relation between the nonlocally correlating events are actually and exclusively determined. In other Lorentz frames, the quantum nonlocal influences 
will no longer be simultaneous, and the time order and the simultaneous time order in the preferred Lorentz frame satisfy Lorentz transformation relations. The causal relations between the nonlocally correlating events in these frames will no longer accord with their time orders in general, but will be determined by their time orders in the preferred Lorentz frame. This guarantees that causes always come before effects in any Lorentz frame, and there will no longer exist any causal loops for the quantum nonlocal influence and the possible QSC based on such influence.

It should be mentioned that Einstein, the founder of special relativity, also realized the possible limitation of the principle of relativity. He thought[21], "As long as one was convinced that all natural phenomena were capable of representation with the help of classical mechanics, there was no need to doubt the validity of this principle of relativity. But in view of the more recent development of electrodynamics and optics, it became more and more evident that classical mechanics affords an insufficient foundation for the physical description of all natural phenomena. At this juncture the question of the validity of the principle of relativity became ripe for discussion, and it did not appear impossible that the answer to this question might be in the negative."

\section{THE POSSIBLE EXISTENCE OF QSC}

In case of the existence of a preferred Lorentz frame, QSC, which uses the quantum nonlocal influence to transfer information faster than light, will not result in the usual causal loop. This may open the door for realizing QSC. In this section, we will further analyze the relation between quantum nonlocal influence and QSC. Given that the minimum ontology is valid, it will be shown 
that the existence of the quantum nonlocal influence may actually result in the availability of QSC.

We have demonstrated the existence of a preferred Lorentz frame among the infinitely many Lorentz frames due to the existence of the quantum nonlocal influence. Can the preferred Lorentz frame be detected? According to one of the most basic of scientific beliefs, namely the minimum ontology, the preferred Lorentz frame should be detectable in principle if it exists. In the following, we will analyze the measurability of the preferred Lorentz frame, and demonstrate that it may result in the availability of QSC.

Since the existence of a preferred Lorentz frame is required by the existence of the quantum nonlocal influence, its detection should relate to this kind of quantum nonlocal influence. For simplicity but without losing generality, we will analyze the quantum nonlocal influence in usual Bell experiment. In order to detect the preferred Lorentz frame or the velocity of the experiment frame relative to it, we must be able to determine the time order of the nonlocally correlating events in the experiment frame. This means that if we measure particle 1 , we must be able to determine the instants $t_{1}$ and $t_{2}$ of the state changes of particle 1 and 2 resulting from the collapse process in the measurement. If $t_{1}=t_{2}$, we can directly find the preferred Lorentz frame. It is just the experiment frame; if $t_{1} \neq t_{2}$, we can calculate the velocity of the preferred Lorentz frame relative to the experiment frame and thus find it. The formula is $u=c^{2} \triangle t / \triangle x$, where $u$ is the velocity of preferred Lorentz frame relative to the experiment frame, $\triangle t=t_{2}-t_{1}, \triangle x=x_{2}-x_{1}$ is the distance between the measuring devices for particle 1 and $2, c$ is light speed. From the above formula we can see that the instant $t_{2}$ may be earlier than $t_{1}$ or later than $t_{1}$. 
This is determined by the distance between the nonlocally correlating events and the direction of the velocity of the preferred Lorentz frame relative to the experiment frame. For the usual situations where $u \ll c, \Delta t \ll \Delta x / c$ is a very short interval.

Once the instant $t_{2}$ of the state change of particle 2 (resulting from the collapse process) can be determined, we can actually realize QSC. The method can be stated as follows. In the above Bell experiment, we first prepare a large number of entangled particle pairs. Then the sender of the information measures particle 1 in each entangled pair one after the other, and encodes the information in the time intervals between the adjacent measurements. Accordingly, the receiver of the information determines the instant $t_{2}$ of the state change of particle 2 in each pair, and decodes the information from the time intervals. Therefore QSC can be achieved.

We have demonstrated that the measurability of the preferred Lorentz frame will result in the availability of QSC. The above demonstration may also provide some possible means of realizing QSC. The key is to determine the instant of the state change of particle 2 resulting from the collapse process. One way is to directly determine the state change of a single particle. This requires that two given nonorthogonal states can be distinguished. The other way is to determine the state changes of a large number of particles, such as measuring the interference pattern. This requires that the state evolution doesn't maintain the orthogonality between states. In the next section, we will see that these requirements are very important in finding a possible method of realizing QSC. 


\section{A POSSIBLE WAY TO REALIZE QSC}

Given that QSC may exist, seeking its realization may be very natural. In this section, we will argue that the combination of quantum collapse and the consciousness of the observer will permit the observer to distinguish nonorthogonal states in principle. This may provide a possible way to realize QSC.

\subsection{A General Analysis}

When physicists discovered that quantum nonlocal influences exist between the measurements on the particles in the quantum entanglement state[1, 2, 3], it was very natural for them to attempt to use the nonlocal influences to transfer information, i.e. realize QSC. One of the best-known efforts was made by Herbert[22]. He tried to decode the information contained in the quantum nonlocal influences by copying the state of a single particle. Wootters and Zurek[23] soon demonstrated that Herbert's copy method is forbidden by the existing (linear) quantum theory. They concluded that a single quantum can't be cloned. In fact, there exists a more general demonstration proving that the existing quantum theory prevents the use of the quantum nonlocal correlation for QSC. Eberhard[24] and Ghirardi et al[25] had given such demonstrations as early as the 1970s, and others also gave similar general demonstrations such as Busch[26]. One common conclusion within the framework of the existing quantum theory is that an unknown quantum state can't be completely determined, and two arbitrarily given nonorthogonal states can't be distinguished.

In order to find the possible way to realize QSC, we need a complete quantum theory. It is well known that the most serious problem in the existing quantum theory is the measurement problem. The existing quantum theory 
doesn't tell us how and when the measurement result appears. The projection postulate is just a makeshift[9]. In this sense, the existing quantum theory is an incomplete description of reality, even if it is a consistent theory through the expression of the projection postulate as a conditional one. On the other hand, mainly due to the research in quantum cosmology[27, 28], physicists have come to realize that the measurement process does not need to be related to the observer (as the orthodox view requires), but must be taken as a self-acting process of the wave function. Therefore it may be very natural to combine the normal linear evolution with the instantaneous collapse process to form a unified evolution process, where the normal linear evolution and the instantaneous collapse process are only two ideal approximations of the unified evolution process. The resulting theory is well known as revised quantum dynamics or dynamical collapse theory, and has been widely and deeply studied in recent times $[15,30,31,29,32,33,34,35,36,37,38,39,40]$. In revised quantum dynamics the linear evolution equation of the wave function is replaced by a stochastic nonlinear equation. The probability prediction about the measurement results is the same as the Born rule in the existing quantum theory, but the instantaneous collapse process is replaced with a describable and dynamical collapse process. At the present time, even if the last complete theory has not been found, there is one thing certain for the revised quantum dynamics: the collapse process of the wave function is one kind of dynamical process, and it will take a finite time interval to finish.

Another alternative to a complete quantum theory is the many-worlds theory $[42,43,44]$. In the many-worlds theory, the linear Schrödinger equation is taken as the complete description of the evolution of the wave function, and there is 
no collapse of the wave function. The theory asserts that the appearance of a definite measurement result results from an objective environmental decoherence process $[45,46]$. When the decoherence process is finished, the whole world splits. This split means that there is a world for each possible definite measurement result, and the observer perceives the corresponding result for all practical purposes (FAPP). The role of the decoherence process in the many-worlds theory is similar to that of the dynamical collapse process in the revised quantum dynamics. They are both used to solve the measurement problem, and explain how and when the measurement results appear. The existence of such objective dynamical processes is the common characteristic of a complete quantum theory. Our following analysis will only rely on this common characteristic.

For simplicity but without losing generality, as a typical example we will primarily analyze the possibility of realizing QSC using the dynamical collapse process in revised quantum dynamics. The conclusion will be also valid for the many-worlds theory.

\subsection{Quantum Observer}

Although no one has strictly demonstrated that revised quantum dynamics does not permit the existence of QSC, physicists generally think that the conclusion should be the same as that of the existing quantum theory. The reason is that these two theories give the same probability prediction about the usual measurement results. However, this conclusion doesn't consider all possible experimental situations. Consider the usual case where physicists argue from the orthodox position of no-QSC for the situation of revised quantum dynamics. This is equivalent to assuming that the observer (and especially his conscious 
perception) does not intervene before the completion of the dynamical collapse. In other words what the observer identifies is only the definite measurement result, and the observer in a quantum superposition state does not exist. Thus the usual no-QSC demonstration doesn't take into consideration the unusual situation in which the observer directly intervenes in the dynamical collapse process and may in fact exist in a quantum superposition state. Since the dynamical collapse process is an objective process that is not related to the consciousness of the observer, the existence of the special case of superposition of the observer can't be excluded in principle. This means that consciousness is not invoked to produce the dynamical collapse process, and the superposition state of the observer with consciousness may exist.

It is still unclear what the perception of the observer in the superposition state is. Albert analyzed a similar situation in detail[47]. He called such a "quantum observer" John. He concluded that John's perception is not the same as one of the definite perceptions in the superposition state, and noted that the perception may be very strange. As we will see, the realization way of QSC is irrelevant to the concrete perception of the "quantum observer" in a superposed state of definite perceptions.

\subsection{The Distinguishability Of Nonorthogonal States}

In this section, we will show that a conscious being may distinguish the definite perception states and the superposition state of them, and further achieve QSC under some condition. This provides a possible way to realize QSC.

Let $\chi_{1}$ and $\chi_{2}$ be two different definite perception states of a conscious being, and $\chi_{1}+\chi_{2}$ is the superposition state of such definite perception states. We 
assume that the conscious being satisfies the following "QSC condition", i.e. that his perception time for the states $\chi_{1}$ and $\chi_{2}$, which is denoted by $t_{p}$, is shorter than the holding time or dynamical collapse time for the superposition state $\chi_{1}+\chi_{2}$, which is denoted by $t_{c}$, and that the time difference $\Delta t=t_{c}-t_{p}$ is large enough for him to identify. According to the prediction of the revised quantum dynamics, the superposition state $\chi_{1}+\chi_{2}$ will generally collapse to one of the definite perception state $\chi_{1}$ or $\chi_{2}$ with the same probability $1 / 2$. In the following, we will demonstrate that the conscious being can distinguish the definite perception states $\chi_{1}$ or $\chi_{2}$ and the superposition state $\chi_{1}+\chi_{2}$. This conclusion is irrelevant to the concrete collapse process of the superposition state $\chi_{1}+\chi_{2}$.

First, we assume that a definite perception about the superposition state $\chi_{1}+$ $\chi_{2}$ can appear only after a dynamical collapse. This is well-accepted as it is in accordance with one of our basic scientific beliefs, i.e. that our inner perception reflects the real world correctly. Under this assumption, the conscious being can have a definite perception about the state $\chi_{1}$ and $\chi_{2}$ after the perception time $t_{p}$, but only after the collapse time $t_{c}$ can the conscious being have a definite perception about the superposition state $\chi_{1}+\chi_{2}$. When the conscious being satisfies the "QSC condition", he can be conscious of the time difference between $t_{p}$ and $t_{c}$, then he can distinguish the definite perception state $\chi_{1}$ or $\chi_{2}$ and the superposition state $\chi_{1}+\chi_{2}[48]$.

Secondly, we assume that the above well-accepted assumption is not true, i.e. that the conscious being can have a definite perception of the superposition state before the dynamical collapse has completed. We will demonstrate that the conscious being is also able to distinguish the states $\chi_{1}+\chi_{2}$ and $\chi_{1}$ or $\chi_{2}$ 
with non-zero probability.

(1). If the definite perception of the conscious being in the superposed state $\chi_{1}+\chi_{2}$ is neither $\chi_{1}$ nor $\chi_{2}$, then the conscious being can directly distinguish the states $\chi_{1}+\chi_{2}$ and $\chi_{1}$ or $\chi_{2}$. For the latter, the definite perception of the conscious being is $\chi_{1}$ or $\chi_{2}$, but for the superposition state $\chi_{1}+\chi_{2}$, the definite perception of the conscious being is neither $\chi_{1}$ nor $\chi_{2}$.

(2). If the definite perception of the conscious being in the superposed state $\chi_{1}+\chi_{2}$ is $\chi_{1}$, then the conscious being can directly distinguish the states $\chi_{1}+\chi_{2}$ and $\chi_{2}$. For the latter, the definite perception of the conscious being is $\chi_{2}$, but for the superposition state $\chi_{1}+\chi_{2}$, the definite perception of the conscious being is $\chi_{1}$ before the collapse process finishes. Besides, the superposition state $\chi_{1}+\chi_{2}$ will become $\chi_{2}$ with probability $1 / 2$ after the collapse process finishes. Then the definite perception of the conscious being will also change from $\chi_{1}$ to $\chi_{2}$ accordingly. For the state $\chi_{1}$ or $\chi_{2}$, the definite perception of the conscious being has no such change. Thus the conscious being is also able to distinguish the states $\chi_{1}+\chi_{2}$ and $\chi_{1}$ with probability $1 / 2$.

(3). If the definite perception of the conscious being in the superposed state $\chi_{1}+\chi_{2}$ is $\chi_{2}$, the demonstration is similar to that of (2).

(4). If the definite perception of the conscious being in the superposed state $\chi_{1}+\chi_{2}$ is random, i.e. one time it is $\chi_{1}$, another time it is $\chi_{2}$, then the conscious being can still distinguish the states $\chi_{1}+\chi_{2}$ and $\chi_{1}$ or $\chi_{2}$ with nonzero probability. For the latter, the perception of the conscious being does not change. For the superposition state $\chi_{1}+\chi_{2}$, the perception of the conscious being will change from $\chi_{1}$ to $\chi_{2}$ or from $\chi_{2}$ to $\chi_{1}$ with non-zero probability during the collapse process with independent randomness. For example, if the 
definite perception of the conscious being in the superposed state $\chi_{1}+\chi_{2}$ is $\chi_{1}$ before the collapse process finishes, but the superposition state becomes $\chi_{2}$ after the collapse process finishes, then the perception of the conscious being will change from $\chi_{1}$ to $\chi_{2}$. If the definite perception of the conscious being in the superposed state $\chi_{1}+\chi_{2}$ assumes $\chi_{1}$ or $\chi_{2}$ with the same probability $1 / 2$, then the above distinguishing probability will be $1 / 2$.

In one word, we have demonstrated that if the conscious being satisfies the "QSC condition", he is able to distinguish the nonorthogonal states $\chi_{1}+$ $\chi_{2}$ and $\chi_{1}$ or $\chi_{2}$ with non-zero probability. This will directly result in the availability of QSC as denoted in Section 2. It should be stressed that, since the collapse time of a single superposition state is an essentially stochastic variable, which average value is $t_{c}$, the "QSC condition" can be in principle satisfied in some collapse events with non-zero probability. For these stochastic collapse processes, the collapse time of the single superposition state is much longer than the (average) collapse time $t_{c}$ and the perception time $t_{p}$. This provides an essential availability of QSC under the assumption that quantum collapse is a real process.

It should be denoted that the above result is also valid in the many-worlds theory and Bohm's hidden variables theory[49, 50]. Thus the existence of QSC may be a natural result of the combination of quantum and consciousness, or a natural result of the complete theory of nature[51]. In the many-worlds theory, the role of the decoherence process is similar to that of the dynamical collapse process in the revised quantum dynamics. Thus the "QSC condition" for the conscious being will be that his perception time for the states $\chi_{1}$ and $\chi_{2}$ is shorter than the environment-induced decoherence time for the superposition 
state $\chi_{1}+\chi_{2}$, and that the time difference is large enough for him to identify. Since the conscious perception process and environment-induced decoherence process are essentially independent, the "QSC condition" can be satisfied in principle. Once the "QSC condition" is satisfied, the conscious being can also distinguish the nonorthogonal states $\chi_{1}+\chi_{2}$ and $\chi_{1}$ or $\chi_{2}$ and further achieve QSC. The conscious being can have a definite perception about the state $\chi_{1}$ and $\chi_{2}$ after the perception time, but only after the decoherence time can the conscious being have a definite perception about the superposition state $\chi_{1}+\chi_{2}$. In Bohm's hidden variables theory, the conscious system in the superposition state $\chi_{1}+\chi_{2}$ and definite state $\chi_{1}$ or $\chi_{2}$ will have different trajectories. Since the conscious system can be conscious of such difference, he can also distinguish the nonorthogonal states $\chi_{1}+\chi_{2}$ and $\chi_{1}$ or $\chi_{2}$ and achieve QSC.

\subsection{A Possible QSC Scheme}

In this section, we will give a possible scheme of achieving QSC based upon the above analysis. It includes two parts. The first part is how to distinguish the nonorthogonal states. We design a device implementing this function, which is called NSDD (Nonorthogonal States Distinguishing Device). The second part is how to achieve QSC using the hardcore device NSDD.

The implementation scheme of NSDD is as follows. The particles to be identified are photons, and the conscious being in the device can perceive a single photon[57]. Let the input states of the device be the nonorthogonal states $\psi_{A}+\psi_{B}$ or $\psi_{A}-\psi_{B}$ and $\psi_{A}$ or $\psi_{B} . \psi_{A}$ is the state of photon with a certain frequency entering into the perception part of the conscious being from the direction $\mathrm{A}$, which can trigger a definite perception $\chi_{A}$ of the conscious being 
who perceives that the photon arrives from the direction A. $\psi_{B}$ is the state of the photon with the same frequency entering into the perception part of the conscious being from the direction $\mathrm{B}$, which can trigger a definite perception $\chi_{B}$ of the conscious being who perceives that the photon arrives from the direction B. $\psi_{A}+\psi_{B}$ and $\psi_{A}-\psi_{B}$ are the direction superposition states of the states $\psi_{A}$ and $\psi_{B}$ of photon[60]. Let the initial perception state of the conscious being is $\chi_{0}$, then after interaction the corresponding entangled state of the whole system is respectively $\psi_{A} \chi_{A}+\psi_{B} \chi_{B}$ and $\psi_{A} \chi_{A}-\psi_{B} \chi_{B}$ for the input states $\psi_{A}+\psi_{B}$ and $\psi_{A}-\psi_{B}$. The conscious being satisfies the "QSC condition", i.e. his perception time for the definite state $\psi_{A} \chi_{A}$ or $\psi_{B} \chi_{B}$, which is denoted by $t_{p}$, is shorter than the dynamical collapse time for the superposition state $\psi_{A} \chi_{A}+\psi_{B} \chi_{B}$ or $\psi_{A} \chi_{A}-\psi_{B} \chi_{B}$, which is denoted by $t_{c}$, and that the time difference $\triangle t=t_{c}-t_{p}$ is large enough for him to identify[61]. According to the above analysis, the device NSDD can distinguish the nonorthogonal states $\psi_{A}+\psi_{B}$ or $\psi_{A}-\psi_{B}$ and $\psi_{A}$ or $\psi_{B}$. When the input state is $\psi_{A}$ or $\psi_{B}$, the conscious being will perceive that the photon arrives from the direction A or B after the perception time $t_{p}$, and he assigns ' 1 ' as the output of the device. When the input state is $\psi_{A}+\psi_{B}$ or $\psi_{A}-\psi_{B}$, the conscious being will perceive that the photon arrives from the direction $\mathrm{A}$ or $\mathrm{B}$ after the collapse time $t_{c}$, and he assigns ' 0 ' as the output of the device. NSDD can be implemented through the direct use of a conscious being or by an advanced consciousness simulation device in the future.

Now we will give the scheme of achieving QSC using the device NSDD. In reality, once the nonorthogonal photon states can be distinguished, achieving QSC will be an easy task, and it may be implemented by means of existing 
technology. Here we use the EPR polarization correlation pairs of photons as the carriers of information. We encode the outgoing information by operating the polarizer, and decode the incoming information using the device NSDD. The experimental setting is shown in Fig.1. Pairs of photons, whose frequencies are $\nu_{1}$ and $\nu_{2}$, are emitted in the $-\mathrm{z}$ direction and $+\mathrm{z}$ direction from a source, are then analyzed by the two-channel polarizers $\pi_{1}$ and $\pi_{2}$ respectively. The optical switch $C_{1}$ in the left side can be controlled to determine whether or not the photon $\nu_{1}$ will pass to $\pi_{1}$. The transmission axes of the polarizers are both set in the direction $\mathrm{x}$. The polarizers $\pi_{1}$ and $\pi_{2}$ allow the polarization components of the photon both parallel to and perpendicular to the transmission axis of the polarizer to be passed. The photon passed and analyzed by the polarizer $\pi_{1}$ is detected by $D_{1}$ or $D_{2}$, and the photon analyzed by the two-channel polarizers $\pi_{2}$ is divided into two paths in space, and respectively input to NSDD from different directions.

We now explain how QSC can be achieved by means of the above setting. Let the sender operate the optical switch $C_{1}$, and have the receiver observe the output of NSDD. Suppose the communication rules are stated as follows. The encoding rule for the sender is that not measuring the photon represents sending the code ' 0 ', and measuring the photon represents sending the code ' 1 '. The decoding rule for the receiver is that the output of NSDD being ' 0 ' represents having received the code '0', and the output of NSDD being '1' represents having received the code ' 1 '.

The communication process can be stated as follows. When the sender wants to send a code ' 0 ', he controls the optical switch $C_{1}$ to let the photon $\nu_{1}$ move freely and not be analyzed by the polarizer $\pi_{1}$. Then the state of the photon $\nu_{2}$ 
is a superposition state like $\psi_{A}+\psi_{B}$ or $\psi_{A}-\psi_{B}$ after it passes the polarizer $\pi_{2}$, and the output of NSDD is ' 0 '. The receiver can decode the sent code as ' 0 '. When the sender wants to send a code ' 1 ', he controls the optical switch $C_{1}$ to allow the photon $\nu_{1}$ to be analyzed by the polarizer $\pi_{1}$ and detected by $D_{1}$ or $D_{2}$ before the photon $\nu_{2}$ arrives at NSDD. Then the state of the photon $\nu_{2}$ collapses to a definite state like $\psi_{A}$ or $\psi_{B}$, and the output of NSDD is ' 1 '. The receiver can decode the sent code as ' 1 '. Thus the sender and receiver can achieve QSC using the above setting and communication rules.

\section{FURTHER ANALYSIS}

In order to further understand the above realization way of QSC, we will give a brief analysis about the relation between quantum collapse and consciousness, and discuss some implications of the existence of QSC.

Bohr first stressed the special role of measurement in quantum theory with his complementarity principle[62]. Later von Neumann rigorously formulated the measurement process mathematically by means of the projection postulate [63], but the inherent vagueness in the definition of a measurement or projection still exists. In order to explain how a definite result is generated by the measurement of an indefinite quantum superposition state, the consciousness of the observer was invoked by von Neumann[63]. This theory was further advocated by Wigner[64], according to which consciousness can break the linear superposition law of quantum mechanics. This may be the first statement made about the relationship between consciousness and collapse. It states that consciousness results in the collapse of wave function.

However as this relationship between the quantum and consciousness needs 
to be greatly revised when faced with the problem of quantum cosmology $[27,28]$. For the state of the whole universe, no outside measuring device or observer exists. Thus the special role of measurement or observation is essentially deprived, and the collapse process, if it exists, must be added to the wave function. The revised quantum dynamics or dynamical collapse theory further revised the above relationship. In the dynamical collapse theory the normal linear evolution and collapse process of the wave function are unified in a stochastic nonlinear Schrödinger equation, and the collapse process is a natural result of such evolution. Thus the new relationship between consciousness and collapse is that collapse of wave function must happen independent of consciousness.

Although the collapse of wave function does not need to resort to the consciousness of an observer, their combination may result in some new results such as the availability of QSC. As the seeds of QSC, consciousness and collapse are both indispensable. Collapse provides the basis, and consciousness provides the means. Even if consciousness doesn't intervene, collapse itself can also display quantum nonlocality, and thus result in the existence of a preferred Lorentz frame. This may further imply the existence of QSC when combined with the minimum ontology. However, collapse alone can't provide the means of realizing QSC, and its inherent randomicity ruthlessly block the way. Here consciousness becomes a delicate bridge to QSC. The direct intervention of consciousness can help to obtain more information about the measured quantum state, which is enough to distinguish nonorthogonal states, and decode the veiled information nonlocally transferred by collapse. This provides a possible way to realize QSC.

Furthermore, it can be seen that the distinguishability of nonorthogonal states will result in the violation of the quantum superposition principle. This 
indicates that consciousness will introduce a new kind of non-linearity to the complete evolution of wave function. The new non-linearity is definite, not stochastic. As we know, nonlinear quantum theory generally has some internal difficulties[65, 66, 67, 68]. For example, the description of composite systems depends on a particular basis in a Hilbert space. However, as we have demonstrated, the consciousness of observer will naturally select a priviliged basis in its state space. Thus the nonlinear quantum evolution introduced by consciousness is logically consistent and may exist[68]. On the other hand, once there exists nonlinear quantum evolution of wave function and real quantum collapse process, the QSC must exist[66, 68]. The reason is that nonlinear evolution doesn't conserve scalar products. States that are initially orthogonal will lose their orthogonality during the evolution. This is also consistent with the above realization way of QSC.

Certainly, once QSC can be realized, we can directly detect the preferred Lorentz frame using the QSC process as stated in Section 2. In fact, some dynamical collapse theories $[15,33,35,36,38]$ also provide a method to detect the preferred Lorentz frame[69]. According to the theories, the collapse time of wave function relates to the velocity of the experimental frame relative to the preferred Lorentz frame. Then the preferred Lorentz frame can be detected through measuring the collapse time of wave function. Besides, QSC can be used as a natural method to synchronize the clocks in different positions, and the simultaneity can be uniquely defined using such superluminal signal[20]. Especially, the one-way speed of light can also be measured. Based on the superluminal synchrony method, the space-time transformation will be not the usual Lorentz transformation, but one kind of superluminal space-time transformation, which 
hold the absoluteness of simultaneity[20]. This may have some implication for the space-time structure and the final combination of quantum and gravity.

Finally, it should be also denoted that the above QSC analysis may provide a physical method of testing the existence of consciousness[70, 71]. We can test whether the conner possesses consciousness through its identification of nonorthogonal states. The conner with consciousness can distinguish the nonorthogonal states, whereas the conner without consciousness can not. This provides a physical way to distinguish between man and machine, and will partially solve one of the hard problems about consciousness, namely 'Who can be said to be a conscious being?'. Besides, the above result may also have some implications for the nature of consciousness. It may imply that consciousness is not reducible or emergent, but a new fundamental property of matter[71]. It is expected that a complete theory of matter must describe all properties of matter, thus consciousness, the new fundamental property of matter, must enter the theory from the start.

Acknowledgments I am very grateful to Gary S. Bekkum for improving the English, and making the text read more smoothly. I am also grateful for the constructive input received from the referees of this journal.

\section{References}

[1] A. Einstein, B. Podolsky, and N. Rosen, Phys. Rev. 47, 777-780 (1935).

[2] J. S. Bell, Physics 1, 195 (1964).

[3] A. Aspect, J. Dalibard, and G. Roger, Phys. Rev. Lett. 49, 1904 (1982). 
[4] G. Weihs, T. Jennewein, C. Simon, H. Weinfurter, and A. Zeilinger, Phys. Rev. Lett. 81, 5039-5043 (1998).

[5] W. Tittel, J. Brendel, N. Gisin, and H. Zbinden, Phys. Rev. A 59, 41504163 (1999).

[6] J. W. Pan, D. Bouwmeester, M. Daniell, H. Weinfurter, and A. Zeilinger, Nature, 403, 515-519 (2000).

[7] A. Shimony, in Quantum Concepts in Space and Time, P. Penrose and C. Isham, ed. (Claredon Press, Oxford, 1996), pp 182.

[8] Y. Aharonov and D. Z. Albert, Phys. Rev. D 24359 (1981).

[9] J. S. Bell, in The Ghost In the Atom, P. Davis and J. Brown, ed. (Cambridge University Press, Cambridge, 1993).

[10] L. Hardy, Phys. Rev. Lett. 68, 2981-2984 (1992).

[11] E. J. Squires, Phys. Lett. A 163, 356-358 (1992).

[12] I. Percival, Phys. Lett. A 244, 495-501 (1998).

[13] I. Percival, LANL e-print quant-ph/9906005 (1999).

[14] Gao Shan, LANL e-print quant-ph/9906113 (1999).

[15] Gao Shan, Quantum Motion and Superluminal Communication, (Chinese BT Press, Beijing, 2000).

[16] G. Auletta, Foundations and Interpretation of Quantum Mechanics, (World Scientific, Singapore, 2000). 
[17] It should be denoted that we can also stipulate that the simultaneity of the collapse of wave function possesses Lorentz invariance. This convention holds the absoluteness of simultaneity, and may have some advantages over the standard convention[18, 19, 20].

[18] Chang T, J. Phys. A, 12, L203 (1979).

[19] P. Caban and J. Rembielinski, Phys. Rev. A 59, 4187-4196 (1999).

[20] Rui Qi, LANL e-print quant-ph/0210021 (2002).

[21] A. Einstein, Relativity: The Special and The General Theory, 1916, 1920, 1952('54).

[22] N. Herbert, Found. Phys. 12, 1171 (1982).

[23] W. K. Wootters and W. H. Zurek, Nature 299, 802 (1982).

[24] P. H. Eberhard, Nuovo Cimento B 46, 392 (1978)

[25] G. C. Ghirardi, A. Rimini, and T. Weber, Letters Nuovo Cimento 27, 293 (1980).

[26] P. Busch, LANL e-print quant-ph/9604014 (1996).

[27] B. S. DeWitt, Phys. Rev. 160, 1113 (1967).

[28] J. B. Hartle and S. W. Hawking, Phys. Rev. 28, 2960 (1983).

[29] G. C. Ghirardi, A. Rimini and T. Weber, Phys. Rev. D 34 470-491 (1986).

[30] P. Pearle, Phys. Rev. A 39, 2277- 2289 (1989).

[31] L. Diosi, Phys. Rev. A, 40, 1165-1174 (1989). 
[32] G. C. Ghirardi, P. Pearle, and A. Rimini, Phys. Rev. A 42 78-89 (1990).

[33] I. C. Percival, Proc. Roy. Soc. Lond. A 447, 189-209 (1994).

[34] R. Penrose, Gen. Rel. and Grav. 28, 581-600 (1996).

[35] L. P. Hughston, Proc. Roy. Soc. Lond. A 452, 953 (1996).

[36] D. I. Fivel, LANL e-print quant-ph/9710042 (1997).

[37] S. L. Adler and T. A. Brun, J. Phys. A 34, 4797-4809 (2001).

[38] Gao Shan, Physics Essays 14 (1), 37-48 (2001).

[39] Gao Shan, Quantum, (Tsinghua University Press, Beijing, 2003).

[40] In $[15,38,39]$, a theory of discontinuous motion of particles is presented. The analysis implies that what quantum mechanics describes is discontinuous motion of particles. The logical basis of discontinuous motion is also given $[39,41]$.

[41] Gao Shan, LANL e-print physics/0209015 (2002).

[42] H. Everett, Rev. Mod. Phys. 29, 454-462 (1957).

[43] B. S. DeWitt and N. Graham ed. The Many-Worlds Interpretation of Quantum Mechanics, (Princeton University Press, Princeton, 1973).

[44] D. Deutsch, Int. J. Theo. Phys. 24, 1-41 (1985).

[45] D. Guilini, E. Joos, C. Kiefer, J. Kupsch, I. O. Stamatiscu, and H. D. Zeh, Decoherence and the Appearance of a Classical World in Quantum Theory, (Springer-Verlag, Berlin, New York, 1996).

[46] W. H. Zurek, Phil. Trans. Roy. Soc. Lond. A 356, 1793-1820 (1998). 
[47] D. Albert, Quantum Mechanics and Experience, (Harvard University Press, Cambridge, 1992).

[48] It should be denoted that Squires also noticed the relationship between the explicit collapse and superluminal signaling under the well-accepted assumption from a slightly different point of view[11].

[49] D. Bohm, Phys. Rev. 85, 166-193 (1952).

[50] D. Bohm and B. J. Hiley, The Undivided Universe: an ontological interpretation of quantum theory, (Routledge, London, 1993).

[51] Some possible evidences have indicated that the human beings in some special states may satisfy the "QSC condition" and achieve QSC[52, 53, $54,55,56]$.

[52] T. D. Duane and T. Behrendt, Science 150, 367 (1965).

[53] R. Targ and H. Puthoff, Nature 252, 602-607 (1974).

[54] D. I. Radin and R. D. Nelson, Found. Phys. 19(12), 1499-1514 (1989).

[55] J. Grinberg-Zylberbaum, D. Dalaflor, L. Attie, and A.Goswami, Physics Essays 7, 422 (1994).

[56] J. Wackermann, C. Seiter, H. Keibel, and H. Walach, Neuroscience Letters 336, 60-64 (2003).

[57] In practical situation, a few photons may be needed[58, 59]. This doesn't influence the scheme.

[58] F. Rieke and D. A. Baylor, Rev. Mod. Phys. 70, 1027 (1998).

[59] F. H. Thaheld, Phys. Lett. A 273, 232-234 (2000). 
[60] G. Ghirardi, Phys. Lett. A 262, 1-14 (1999).

[61] In real experiments, conscious perception may be more accurately described by the EEG recording of the conscious being, and the "QSC condition" can also be strictly stated using the corresponding EEG recording.

[62] N. Bohr, Nature 121, 580-590 (1927).

[63] John von Neumann, Mathematical Foundations of Quantum Mechanics, (Princeton University Press, Princeton, 1955).

[64] E. P. Wigner, Symmetries and Reflections, (Indiana University Press, Bloomington and London, 1967), pp 171-184.

[65] S. Weinberg, Dreams of a Final Theory, (Pantheon Books, New York, 1992).

[66] N. Gisin, Phys. Lett. A 143, 1-2 (1990)

[67] J. Polchinski, Phys. Rev. Lett. 66, 397 (1991).

[68] M. Czachor, LANL e-print quant-ph/9501007 (1995)

[69] Gao Shan, CERN preprint EXT-2003-061 (2003).

[70] Gao Shan, The Noetic Journal 3(3), 233-235 (2002).

[71] Gao Shan, NeuroQuantology 1, 4-9 (2003). 


\section{Figures Captions}

Fig.1 The setting of a possible QSC Scheme 


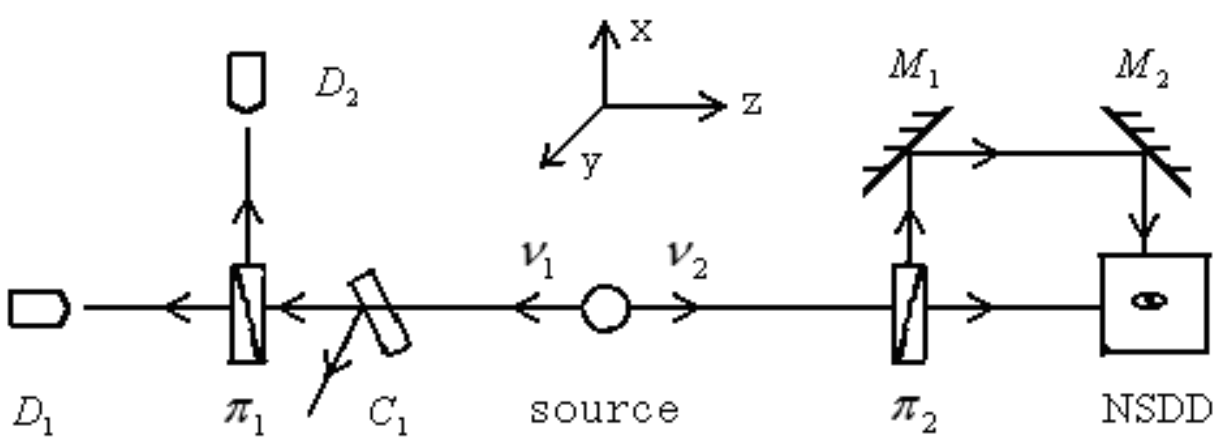

